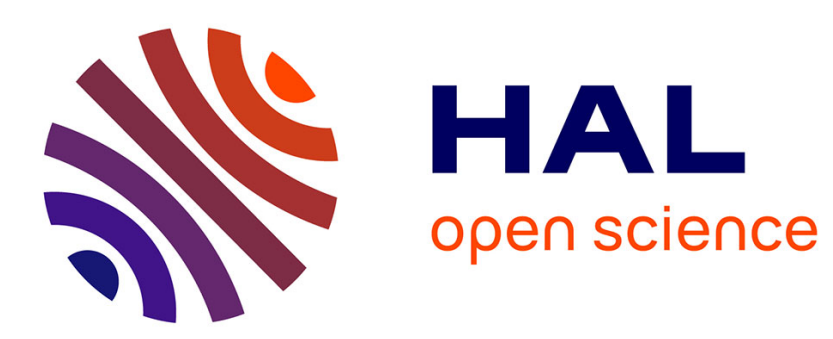

\title{
Generalized characteristics of resonant polymer-coated microcantilevers in viscous liquid media
}

Russel Cox, Michael Wenzel, Fabien Josse, Stephen Heinrich, Isabelle Dufour

\section{To cite this version:}

Russel Cox, Michael Wenzel, Fabien Josse, Stephen Heinrich, Isabelle Dufour. Generalized characteristics of resonant polymer-coated microcantilevers in viscous liquid media. IEEE Frequency Control Meeting, 2007, Genève, Switzerland. pp.6. hal-00181952

\section{HAL Id: hal-00181952 https://hal.science/hal-00181952}

Submitted on 6 Nov 2007

HAL is a multi-disciplinary open access archive for the deposit and dissemination of scientific research documents, whether they are published or not. The documents may come from teaching and research institutions in France or abroad, or from public or private research centers.
L'archive ouverte pluridisciplinaire HAL, est destinée au dépôt et à la diffusion de documents scientifiques de niveau recherche, publiés ou non, émanant des établissements d'enseignement et de recherche français ou étrangers, des laboratoires publics ou privés. 


\section{Generalized Characteristics of Resonant Polymer- Coated Microcantilevers in Viscous Liquid Media}

\author{
R. Cox, M. J. Wenzel, F. Josse, S. M. Heinrich ${ }^{1}$ \\ Department of Electrical and Computer Engineering, \\ ${ }^{1}$ Civil and Environmental Engineering \\ Marquette University \\ Milwaukee, WI, USA
}

\author{
I. Dufour \\ Université Bordeaux 1 \\ CNRS, IMS Laboratory \\ Talence, France
}

\begin{abstract}
Expressions describing the resonant frequency and quality factor of a dynamically-driven, polymer-coated microcantilever in a viscous liquid medium have been obtained. These generalized formulas are used to describe the effects the operational medium and the coating has on the device sensitivity when used in liquid-phase chemical sensing applications. The derived expressions are shown to reduce to well-known formulas for the case of an uncoated cantilever in an invisicid medium and the case of a coated cantilever in air. The theoretical results are compared to existing chemical sensor data in aqueous and viscous solutions.
\end{abstract}

\section{INTRODUCTION}

Polymer-coated microcantilevers have been extensively investigated for use as chemical sensor platforms [1-4]. Microcantilevers have shown high sensitivities in chemical vapor detection due to their high surface area to mass ratio. Application of microcantilevers to liquid-phase detection has mostly focused on static mode detection because dynamicallydriven microcantilevers suffer from low frequency stability in viscous media [5]. The characteristics of uncoated dynamically-driven microcantilevers have been previously investigated in viscous media [6]. Recently, work has been done on characterizing the behavior of polymer-coated microcantilevers in a vacuum, which indicated significant chemically induced coating plasticization effects [1]. However, the effects of the polymer coating in a viscous liquid environment have not been studied. The present derivation extends previous work on uncoated and coated microcantilevers to obtain generalized formulas for the characteristics of a polymer-coated microcantilever operating in a viscous liquid medium.

\section{THEORY}

\section{A. Generalized Equation of Motion}

Figure 1 shows the length, $L$, width, $b$, and thickness, $h_{l}$, of the microcantilever as well as the polymer layer's thickness, $h_{2}$. The deflection function, $w(x, t)$, which represents the vertical displacement along the length of the beam as a function of time is also indicated.
Assuming that the microcantilever is a rectangular beam with $L>>b, \quad b>h_{1}$ and $\mathrm{b}>h_{2}$, undergoing small lateral deformations, the well-known equation of motion for an uncoated microcantilever operating in a vacuum is given by

$$
E I \frac{\partial^{4} w(x, t)}{\partial x^{4}}+m_{B} \frac{\partial^{2} w(x, t)}{\partial t^{2}}=F(x) e^{j \omega t},
$$

where $E I$ and $m_{B}$ are the beam's flexural rigidity and mass per unit length, respectively, and $F(x)$ is the position dependent forcing function per unit length operating at a angular frequency of $\omega$ [1]. Using the modifications to the equation of motion found in [1] and [6], a generalized equation of motion for a polymer-coated microcantilever in an unbounded, viscous liquid medium is given by

$$
(E I) \frac{\partial^{4} w(x, t)}{\partial x^{4}}+m_{B} * \frac{\partial^{2} w(x, t)}{\partial t^{2}}=F(x) e^{j \omega t},
$$

where $m_{B}{ }^{*}$ is the effective mass per unit length and the flexural rigidity of the polymer-coated microcantilever is [1]

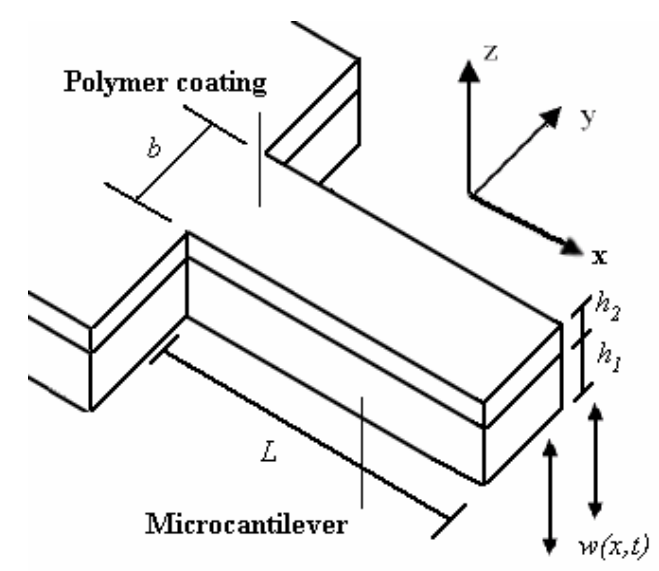

Figure 1: A rectangular microcantilever shown with a polymer layer 


$$
(E I)^{*}=\left(\sqrt{(E I)^{\prime 2}+(E I)^{\prime 2}}\right) e^{j \theta}
$$

with

$$
(E I)^{\prime}=\left(E_{1} I_{1}+E_{2}^{\prime} I_{2}\right),
$$

and

$$
(E I)^{\prime \prime}=\left(E_{2}^{\prime \prime} I_{2}\right)
$$

$E_{1}$ is the Young's modulus of the base layer, $E_{2}{ }_{2}$ and $E_{2}{ }^{\prime \prime}$ are the storage modulus and loss modulus of the polymer layer, respectively, and $I_{1}$ and $I_{2}$ are the moment of inertia of the base and polymer layer, respectively. For a system with a polymer layer, the moment of inertia can be calculated using a time-invariant neutral axis approximation [1]. For composite base-layer microcantilevers, such as a CMOS based cantilever, the flexural rigidity can be found by replacing $E_{l} I_{1}$ in eq. (4) with the sum of each layer's flexural rigidity [7]. $\theta$ in eq. (3) is the composite loss angle of the beam, and is defined as

$$
\theta=\tan ^{-1}\left(\frac{h_{2} E_{2}^{\prime \prime}(\omega)}{h_{1} E_{1}+h_{2} E_{2}^{\prime}(\omega)}\right) .
$$

Here, the dependence of $E_{2}^{\prime}$ and $E_{2}^{\prime \prime}$ on the operating frequency has been explicitly stated.

The effective mass per unit length, $m_{B}{ }^{*}$, accounts for both the mass of the coated-cantilever and the displaced mass of the fluid, as well as the viscous damping caused by fluid shear near the edges of the microcantilever [8]. The viscous damping is represented by $g_{l}$, given by

$$
g_{1}=\frac{\pi}{4} \rho_{L} b^{2} \Gamma_{i}(R e) \omega,
$$

and the mass of the displaced fluid is represented by $g_{2}$, given by [9]

$$
g_{2}=\frac{\pi}{4} \rho_{L} b^{2} \Gamma_{r}(R e) .
$$

Here, $\rho_{L}$ is the density of the medium and $\Gamma_{\mathrm{r}}(R e)$ and $\Gamma_{\mathrm{i}}(R e)$ are the real and imaginary components of the hydrodynamic function, $\Gamma(R e)$, of the microcantilever, given by

$$
\Gamma(R e)=\Omega(R e)\left(1+\frac{4 j K_{1}(-j \sqrt{j R e})}{\sqrt{j \operatorname{Re}} K_{0}(-j \sqrt{j R e})}\right) .
$$

In eq. (9), $\Omega(R e)$ is the function used to correct the hydrodynamic function of a beam of circular cross section, $\Gamma_{\text {circ }}(R e)$, to that of a rectangular beam [6]. $K_{0}$ and $K_{l}$ are modified Bessel functions. The hydrodynamic function is dependent on the Reynolds number [8], which is a measure of the ratio of inertial forces to the viscous forces acting on the beam, and is defined as

$$
R e=\frac{\rho_{L} \omega b^{2}}{4 \eta_{L}},
$$

where $\eta_{\mathrm{L}}$ is the viscosity of the fluid. The force exerted by the fluid is a sum of the viscous force and the inertial forces,

$$
F_{\text {fluid }}(x, t)=-g_{1} \frac{\partial w(x, t)}{\partial t}-g_{2} \frac{\partial^{2} w(x, t)}{\partial t^{2}} .
$$

Assuming a sinusoidal deflection function, eq. (11) can be converted to fit the form of eq. (2), resulting in the effective mass per unit length given by

$$
m_{B}^{*}=\sqrt{\left(m / L+g_{2}\right)^{2}+\left(g_{1} / \omega\right)^{2}} e^{j \phi},
$$

where

$$
\phi=-\tan ^{-1}\left(\frac{L\left(g_{1} / \omega\right)}{m+L g_{2}}\right) .
$$

and $m$ is the mass of the microcantilever with the coating.

\section{B. Generalized Resonant Frequency}

The resonant frequency of a coated-microcantilever in a viscous medium can be found using the generalized equation of motion, eq. (2). It is commonly assumed that the Young's modulus of the base layer is frequency-independent. For the polymer layer, the Young's modulus depends on the frequency. However, a first approximation can be taken equal to its value taken at the operating resonant frequency. The Reynolds number of the system is linearly proportional to the resonant frequency. This dependence must be considered whenever the viscous forces are significant (i.e., relatively small Reynolds numbers). Assuming a frequencydependent hydrodynamic function, the resonant frequency obtained by solving eq. (2) is given by

$$
f_{\text {res }, i}=\frac{\alpha_{i}^{2}}{2 \pi L^{2}} \sqrt{\frac{K}{M}}
$$

where $\alpha_{i}$ is the $i$ th root of

$$
\begin{gathered}
\cos \left(\alpha_{i}\right) \cosh \left(\alpha_{i}\right)-1=0, \\
K=\left(m / L+g_{2}+\left(\frac{\omega}{2}\right) \frac{d}{d \omega}\left(g_{2}\right)\right)(E I)^{\prime} \\
-\left(\left(g_{1} / \omega\right)+\left(\frac{\omega}{2}\right) \frac{d}{d \omega}\left(g_{1} / \omega\right)\right)(E I)^{\prime \prime}, \\
M=\left(\left(g_{1} / \omega\right)+\left(\frac{\omega}{2}\right) \frac{d}{d \omega}\left(g_{1} / \omega\right)\right)\left(g_{1} / \omega\right) \\
+\left(m / L+g_{2}+\left(\frac{\omega}{2}\right) \frac{d}{d \omega}\left(g_{2}\right)\right)\left(m / L+g_{2}\right),
\end{gathered}
$$




$$
\begin{aligned}
& \frac{d}{d \omega}\left(g_{1} / \omega\right)=\frac{\pi}{4} \rho_{L} b^{2} \Lambda_{i}(R e), \\
& \frac{d}{d \omega}\left(g_{2}\right)=\frac{\pi}{4} \rho_{L} b^{2} \Lambda_{r}(R e),
\end{aligned}
$$

and $\Lambda_{r}(R e)$ and $\Lambda_{i}(R e)$ are the real and imaginary components of $\Lambda(R e)$, which is the defined as the derivative of the hydrodynamic function. This function can be obtained as

$$
\begin{aligned}
& \Lambda(R e)=2 \Omega(R e)\left(\frac{\left(K_{1}(-j \sqrt{j R e})\right)^{2}-K_{2}(-j \sqrt{j R e})\left(K_{0}(-j \sqrt{j R e})\right)}{\omega\left(K_{0}(-j \sqrt{j R e})\right)^{2}}\right) \\
& +\Gamma_{\text {circ }}(R e) \frac{d}{d \omega}(\Omega(R e)) .
\end{aligned}
$$

$\Lambda(R e)$ is only dependent on the Reynolds number since $\Omega(R e)$ also has a derivative inversely proportional to $\omega$. Through the use of an iterative process, eq. (14) can be solved. Iterative correction uses an initial guess for $\omega$ (for example, the resonant frequency in a vacuum). A value for the Reynolds number can then be calculated, which in turn can be used to update the value of the $\omega$.

If both the Young's modulus of the polymer layer and the Reynolds number are assumed frequency-independent near resonance, the resonant frequency can be simplified to

$$
f_{\text {res }, i}=\frac{\alpha_{i}^{2}}{2 \pi L^{2}} \sqrt{\frac{\left(m / L+g_{2}\right)(E I)^{\prime}-\left(g_{1} / \omega\right)(E I)^{\prime \prime}}{\left(m / L+g_{2}\right)^{2}+\left(g_{1} / \omega\right)^{2}}} .
$$

It can be shown that both eq. (14) and eq. (21) reduce to the well-known expression for the resonant frequency of a coated-microcantilever in a vacuum $\left(\mathrm{g}_{1}=\mathrm{g}_{2}=0\right)$ [2], given by

$$
f_{0}=\frac{\alpha_{0}{ }^{2}}{2 \pi L^{3 / 2}} \sqrt{\frac{E_{1} I_{1}+E_{2}^{\prime} I_{2}}{m}} .
$$

with $\alpha_{0} \cong 1.875$ corresponding to the fundamental flexural mode.

If there are only small dissipative effects from both the medium and the polymer in a viscous liquid medium, the resonant frequency simplifies to

$$
f_{\text {res }}=f_{0} \frac{1}{\sqrt{1+L g_{2} / m}} .
$$

This is equivalent to the resonant frequency obtained in [6]. When the medium is assumed inviscid, eq. (23) can be further simplified to a form analogous to the one presented in [10] as

$$
f_{\text {res }}=f_{0} \frac{1}{\sqrt{1+L \pi \rho_{L} b^{2} /(4 m)}} .
$$

\section{Quality Factor}

Operation in a viscous liquid medium drastically decreases the frequency stability of microcantilevers in an oscillator configuration. Equation (2) can be used to calculate the quality factor of the system. Because it is difficult to obtain an analytical expression for the quality factor, it is best solved numerically. This is done by calculating the upper and lower $3 \mathrm{~dB}$ frequencies through an iterative process.

If both the Young's modulus of the polymer layer and the Reynolds number are again considered constant in the range of operational frequencies, the quality factor can be obtained from eq. (2) as

$$
Q=\left(2\left(1-\sqrt{\left.1-\mid \frac{\left(g_{1} / \omega\right)(E I)^{\prime}+\left(m / L+g_{2}\right)(E I)^{\prime \prime}}{\left(m / L+g_{2}\right)(E I)^{\prime}-\left(g_{1} / \omega\right)(E I)^{\prime \prime} \mid}\right)}\right)^{-1} .\right.
$$

The above approximation is found to be in good agreement with the numerically derived generalized quality factor for $R e>>1$. Assuming operation in a vacuum, $g_{1}$ and $g_{2}$ are both zero and eq. (25) reduces to the one presented in [1],

$$
Q_{0}=\left(2\left(1-\sqrt{1-\frac{(E I)^{\prime \prime}}{(E I)^{\prime}}}\right)\right)^{-1}
$$

In low loss systems, eq. (25) can further be approximated as

$$
Q_{\text {approx }}=\frac{\left(m / L+g_{2}\right)(E I)^{\prime}-\left(g_{1} / \omega\right)(E I)^{\prime \prime}}{\left(g_{1} / \omega\right)(E I)^{\prime}+\left(m / L+g_{2}\right)(E I)^{\prime \prime}} .
$$

For an uncoated cantilever, eq. (27) reduces to the estimation of the quality factor in low loss from [6]. Other losses can be added into the quality factor by the well-known equation for dealing with multiple loss systems, as

$$
\frac{1}{Q_{\text {sum }}}=\sum_{i} \frac{1}{Q_{i}} .
$$

$Q_{i}$ is the quality factor associated with a particular loss source. In practical applications, the losses caused by the medium and the polymer coating are normally the dominant terms. Using eq. (28), taking only into account these losses, the quality factor can be approximated as

$$
Q_{\text {sum }}=\left(\frac{\left(g_{1} / \omega\right)}{\left(m / L+g_{2}\right)}+\frac{(E I)^{\prime \prime}}{(E I)^{\prime}}\right)^{-1}
$$

When $\left(g_{1} / \omega\right)(E I)^{\prime \prime}$ is negligible, eq. (27) reduces to eq. (29). Under certain conditions, other losses, such as from the squeeze film effect or clamping, can significantly contribute to the decrease of the quality factor [8].

\section{Effects of Chemical Analyte Soption into the Coating}

Introduction of a chemical analyte into the operational medium will not only change the medium's viscosity and 
density, but will also affect the characteristics of the coated microcantilever through chemical sorption into the polymer layer. For example, the density and thickness of the polymer layer will increase; the moment of inertia for both layers will change due to a shift in the neutral axis; and the complex Young's moduli of the polymer layer will change. While these effects on the resonant frequency, $f_{\text {res }}$, are generally assumed negligible, they can be significant in the determination of $\Delta f_{\text {res. }}$. This shift in the resonant frequency in turn can be used to determine the chemical concentration of the analyte in the medium.

The change in the viscosity and density of the solution can be accounted for by differential measurement using an uncoated reference cantilever of the same geometry; therefore, these effects will not be included in the discussion herein. Assuming only small variations in the system, the shift in the resonant frequency can be found as a function of the change in microcantilever mass, $\Delta m$, changes in storage and loss modulus, $\Delta E_{2}^{\prime}$ and $\Delta E_{2}^{\prime \prime}$, and changes in the moments of inertia $\Delta I_{1}$ and $\Delta I_{2}$ as

$\Delta f_{r e s} \cong \lambda_{m} \Delta m+\lambda_{E_{2}}{ }^{\prime} \Delta E_{2}{ }^{\prime}+\lambda_{E_{2}}{ }^{\prime \prime} \Delta E_{2}{ }^{\prime \prime}+\lambda_{I_{1}} \Delta I_{1}+\lambda_{I_{2}} \Delta I_{2}$.

In eq. (30), the $\lambda$ terms describe the sensitivities to various changes in the system, and are given as

$$
\begin{gathered}
\lambda_{m}=\left(\begin{array}{l}
\frac{\left(\left(g_{1} / \omega\right)+\left(\frac{\omega}{2}\right) \frac{d}{d \omega}\left(g_{1} / \omega\right)\right)\left(g_{1} / \omega\right)(E I)^{\prime}}{2 K L M} \\
+\frac{\left(\left(g_{1} / \omega\right)+\left(\frac{\omega}{2}\right) \frac{d}{d \omega}\left(g_{1} / \omega\right)\right)\left(m / L+g_{2}\right)(E I)^{\prime \prime}}{2 K L M} \\
-\frac{\left(m / L+g_{2}+\left(\frac{\omega}{2}\right) \frac{d}{d \omega}\left(g_{2}\right)\right)}{2 L M}
\end{array} f_{\text {res }}\right. \\
\lambda_{E_{2}^{\prime}}=\frac{I_{2}\left(m / L+g_{2}+\left(\frac{\omega}{2}\right) \frac{d}{d \omega}\left(g_{2}\right)\right) f_{\text {res }}}{2 K} \\
\lambda_{E_{2}^{\prime \prime}}=\frac{-I_{2}\left(\left(g_{1} / \omega\right)+\left(\frac{\omega}{2}\right) \frac{d}{d \omega}\left(g_{1} / \omega\right)\right) f_{\text {res }}}{2 K} \\
\lambda_{I_{1}}=\frac{E_{1}\left(m / L+g_{2}+\left(\frac{\omega}{2}\right) \frac{d}{d \omega}\left(g_{2}\right)\right) f_{\text {res }}}{2 K}
\end{gathered}
$$

and

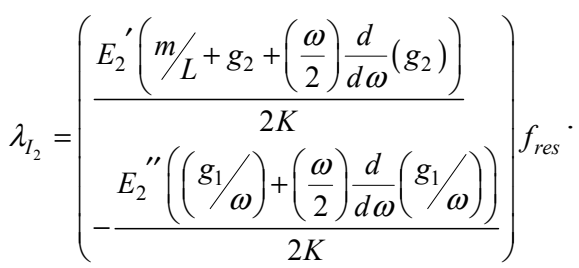

It is noted that the swelling induced change in the polymer thickness is included in the $\Delta I$ terms. All other terms (terms including $\Delta$ products) have been assumed negligible for small frequency shifts. Also, eq. (31d) and eq. (31e) show that shifts in the moment of inertia can be disregarded. This is similar to what was shown in [2]. Equations (30) and (31) can then be used to analyze the sensitivity response of coated microcantilever chemical sensors.

\section{RESULTS}

\section{A. Simulations}

Simulations are performed to illustrate the effects of coating and medium properties for various microcantilever geometries. The polymer layer used in these simulations is polyisobutylene (PIB). The Young's modulus for PIB has been previously characterized in [11]. Figure 2 and 3 shows the frequency response of a polymer-coated microcantilever to different aqueous mixtures of glycerol and ethanol, respectively.

For low Reynolds numbers, Re, i.e. high viscosity fluid and/or low density, the results indicate that the hydrodynamic function cannot be considered frequency-independent. Therefore, $g_{1}$ and $g_{2}$ must be considered frequency dependent in calculating the resonant frequency. Figure 3 indicates that the resonant frequency is not just dependent on the viscosity, but the overall Reynolds number. While ethanol has a higher viscosity compared to water, its higher fluid density leads to an overall higher Reynolds number and resonant frequency.

The calculated quality factor using the upper and lower 3 $\mathrm{dB}$ frequencies and eq. (25) shows a negligible difference in water and glycerol. Figures 4 and 5 compare the calculated quality factor with that of low loss approximation using eq. (27). As expected, eq. (27) is shown to be inappropriate for high viscosity media. The difference between the approximation and the exact calculation is found to increase as the Reynolds number decreases. In the calculations, it is assumed that the Young's modulus of the coating does not change as a function of the operational medium.

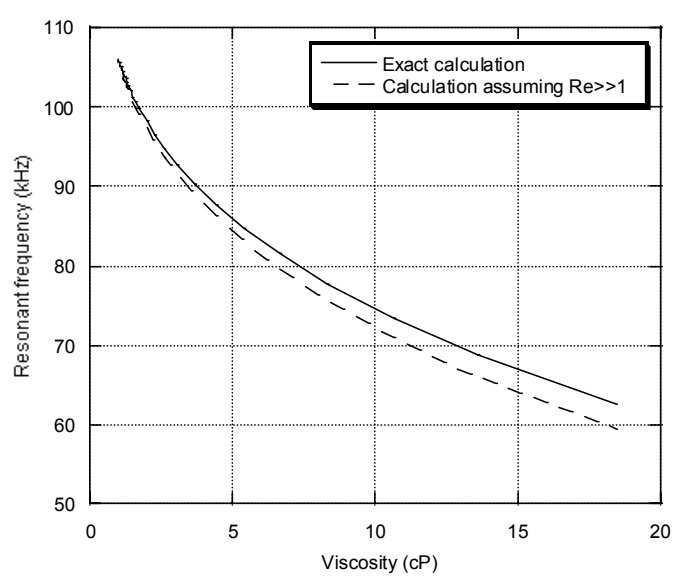

Figure 2: Calculated resonant frequency of a $100 \times 20 \times 2 \mu \mathrm{m}$ microcantilever coated with $0.5 \mu \mathrm{m}$ of PIB in varying mixtures of glycerol (up to $64 \%$, or $18 \mathrm{cP}$ ) 


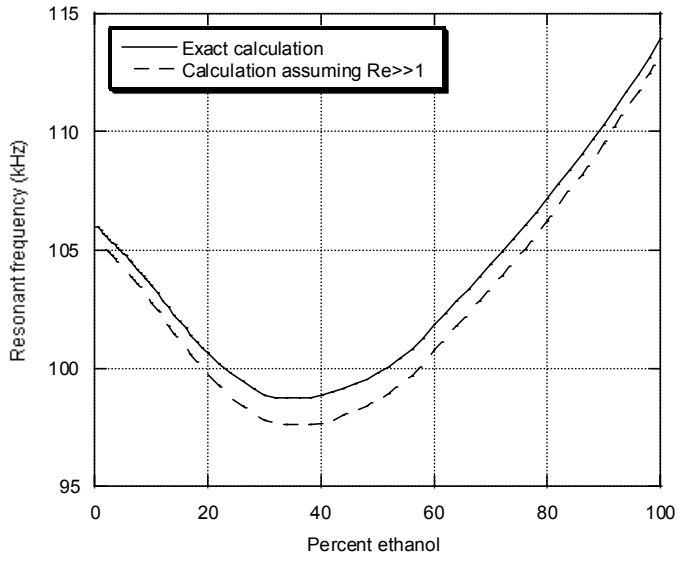

Figure 3: Calculated resonant frequency of a $100 \times 20 \times 2 \mu \mathrm{m}$ microcantilever with a $0.5 \mu \mathrm{m}$ PIB polymer-coating in varying mixtures of ethanol

Chemical absorption into the polymer coating is then simulated assuming an increase of polymer mass by up to $5 \%$ $(45.85 \mathrm{pg})$, a storage modulus decrease by up to $10 \%(-6.73$ $\mathrm{MPa})$, and a loss modulus increase by up to $100 \%(+102.48$ $\mathrm{MPa}$ (Figs. 6 and 7). The mass absorption is found to cause up to a $42 \mathrm{~Hz}$ decrease in the resonant frequency in water, whereas the coating plasticization effects contribute up to an additional $9.9 \mathrm{~Hz}$ decrease in the resonant frequency. In $40 \%$ glycerol $(\sim 3.6 \mathrm{cP})$, the same mass loading causes up to a 23.9 $\mathrm{Hz}$ decrease in the resonant frequency, whereas the coating plasticization effects contribute an additional $11.7 \mathrm{~Hz}$ decrease in the resonant frequency. This indicates that the sensitivity to mass loading decreases in higher viscosities solutions, while the sensitivity to changes in the Young's modulus increases in higher viscosities.

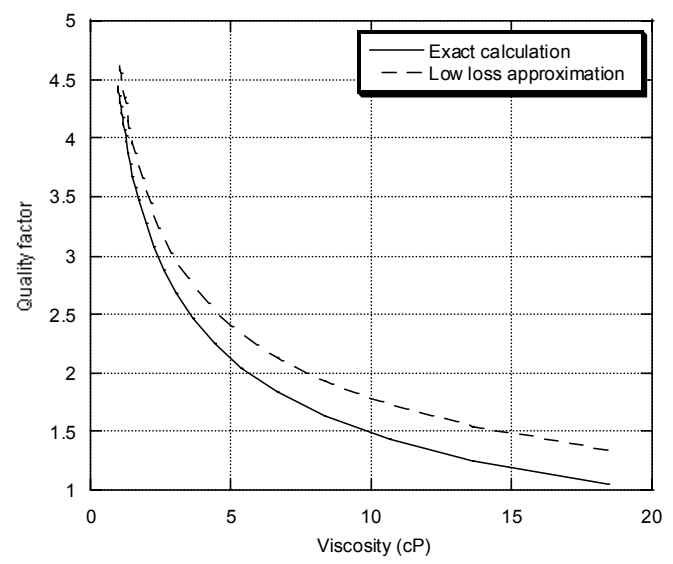

Figure 4: Calculated quality factor of a $100 \times 20 \times 2 \mu \mathrm{m}$ microcantilever with a $0.5 \mu \mathrm{m}$ PIB coating in varying mixtures of glycerol (up to $64 \%$ ) compared with the small loss approximation $Q_{a p p r o x}$

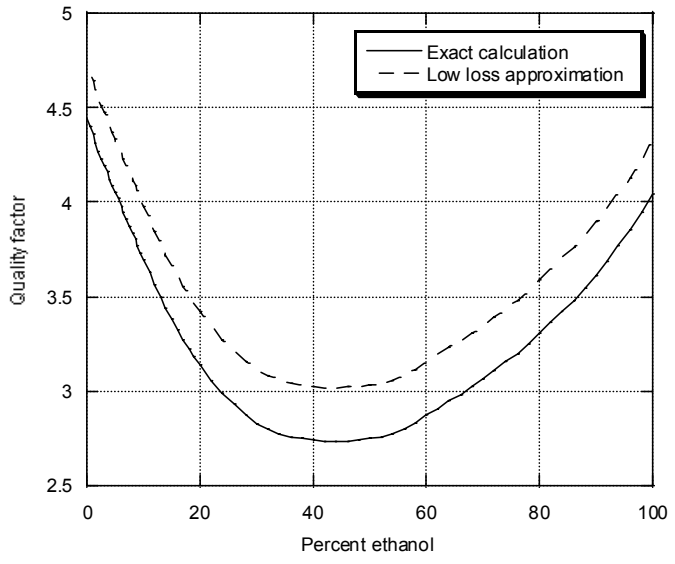

Figure 5: Calculated quality factor of a $100 \times 20 \times 2 \mu \mathrm{m}$ microcantilever with a $0.5 \mu \mathrm{m}$ PIB polymer-coating in varying mixtures of ethanol compared with the small loss approximation $Q_{\text {approx }}$

\section{B. Comparison to Experimental Data}

Recently performed experiments have shown the feasibility of dynamically-driven CMOS multilayer microcantilever chemical sensors in liquid environments [3]. With a reference microcantilever to account for the effects of the medium, the response of a $150 \times 140 \times 8.2 \mu \mathrm{m}$ CMOS-based microcantilever coated with $0.3 \mu \mathrm{m}$ of PIB to $350 \mathrm{ppm}$ ethylbenzene was found to result in a shift in the resonant frequency of about $85 \mathrm{~Hz}$. However, the theoretical model used in [3] assuming only mass loading predicted a $63 \mathrm{~Hz}$ shift in the resonant frequency.

For this cantilever geometry since the condition $L>>b$ is not satisfied, shearing effects can no longer be considered negligible. For the associated fluid properties, the Reynolds number is quite large, i.e. $R e>>1$. Using eq. (23), $g_{2}$ can be obtained as

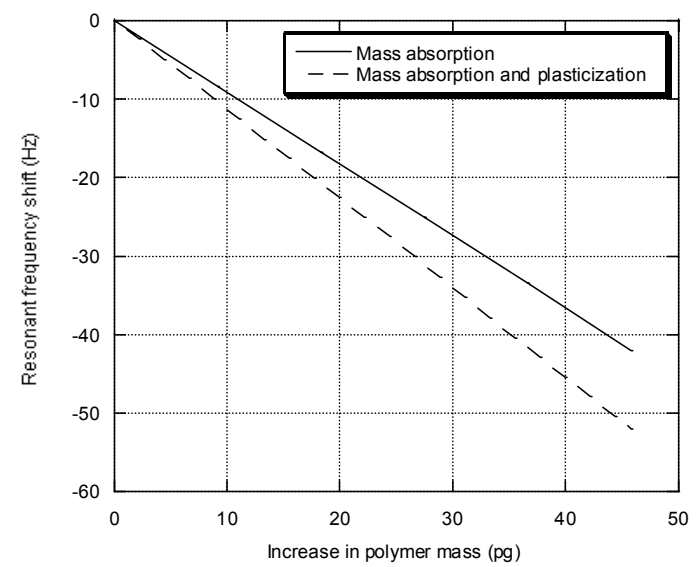

Figure 6: Shifts in the resonant frequency of a $100 \times 20 \times 2 \mu \mathrm{m}$ microcantilever with a $0.5 \mu \mathrm{m}$ PIB coating in water undergoing mass absorption of up to $45 \mathrm{pg}$ with and without coating plasticization effects 


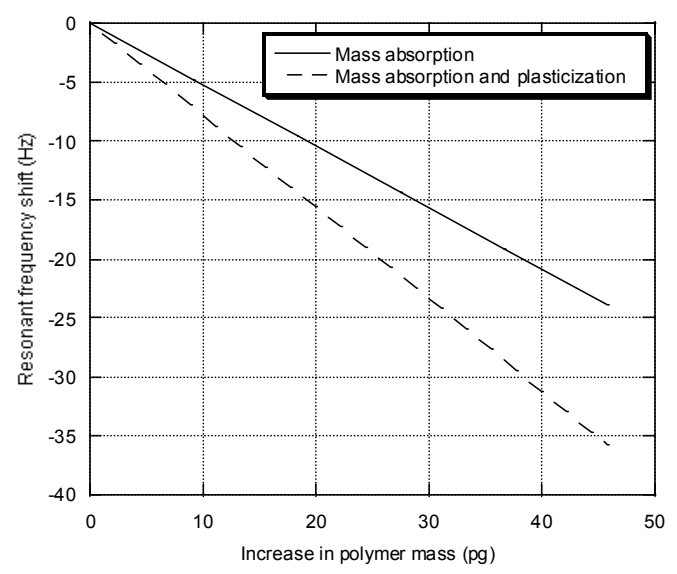

Figure 7: Shift in the resonant frequency of a $100 \times 20 \times 2 \mu \mathrm{m}$ microcantilever with a $0.5 \mu \mathrm{m}$ PIB polymer-coating in a $40 \%$ glycerol mixture undergoing mass absorption of up to $45 \mathrm{pg}$ with and without coating plasticization effects

$$
g_{2}=\left(\left(\frac{f_{0}}{f_{\text {res }}}\right)^{2}-1\right) \mathrm{m} / \mathrm{L} .
$$

Using the measured resonant frequencies, $f_{0}$ and $f_{\text {res }}$, in air and the liquid, respectively, $g_{2}$, can be empirically extracted from eq. (32), and $g_{l}$ can be assumed zero.

Using eq. (30a) and the frequency shift from mass loading, the decrease in the resonant frequency due to mass absorption alone is $63 \mathrm{~Hz}$ at a concentration of $350 \mathrm{ppm}$. Assuming up to a $-70 \%$ decrease in storage modulus and up to a $700 \%$ increase in loss modulus, an additional $7 \mathrm{~Hz}$ decrease in the resonant frequency can be accounted for by coating plasticization (Fig. 8).

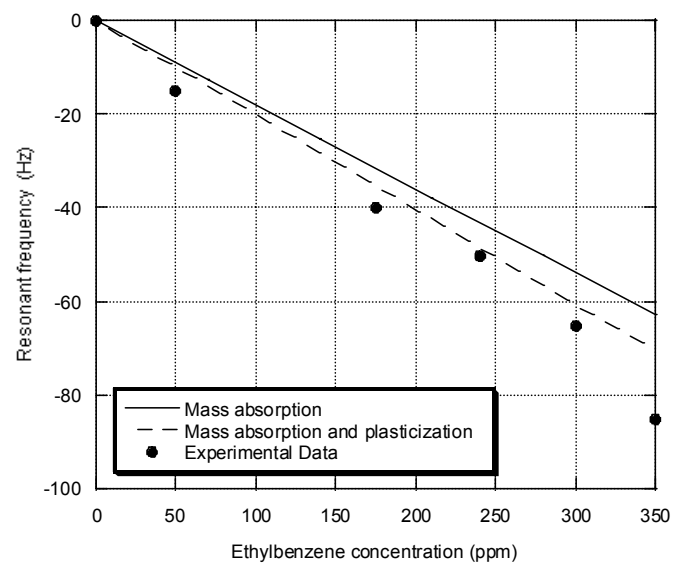

Figure 8: Comparison of experimentally measured values in [3] for the resonant frequency shift of a $150 \times 140 \times 8.2 \mu \mathrm{m}$ microcantilever coated with $0.3 \mu \mathrm{m}$ of PIB to the theoretically calculated frequency shift taking into account first mass absorption and then mass absorption and plasticization effects. Note that a reference cantilever is used in [3] to account for the effects of changes in the medium properties.

\section{CONCLUSIONS}

Generalized equations for the resonant frequency and quality factor of a dynamically driven, polymer-coated microcantilever accounting for the effects of the medium and the polymer coating have been presented. It is found that the resonant frequency and quality factor can be significantly affected by non-mass loading effects. It is also found that the resonant frequency's sensitivity to mass loading and other system properties are dependent on the medium of operation. The generalized equations obtained in this work simplify to known special cases and can be used to estimate the contribution of non-mass absorption effects in the response of dynamically-driven microcantilever chemical sensors.

\section{REFERENCES}

[1] U. Sampath, "Analytical Modeling of Microcantilever-Based Dynamic Microsensors," M.S. Thesis, Marquette University, Milwaukee, WI, 2005.

[2] U. Sampath, S.M. Heinrich, F. Josse, F. Lochon, I. Dufour, D. Rebiere "Study of Viscoelastic Effect on the Frequency Shift of Microcantilever Chemical Sensors," IEEE Transactions on Ultrasonics, Ferroelectrics and Frequency Control, Vol.53, n¹1, 2006, pp. 2166-2173.

[3] C. Vancura, Y. Li, J. Lichtenberg, K.-U. Kirstein, A. Hierlemann, F. Josse, "Liquid-Phase Chemical and Biochemical Detection Using Fully Integrated Magnetically Actuated Complementary Metal Oxide Semiconductor Resonant Cantilever Sensor Systems," Anal. Chem., 79 (4), 2007, pp. $1646-1654$

[4] F. Lochon, L Fadel, I. Dufour, D. Rebiere, J. Pistre, "Silicon made resonant microcantilever: Dependence of the chemical sensing performances on the sensitive coating thickness," Materials Science and Engineering: C, vol 26, Issues 2-3, March 2006, pp. 348-353.

[5] L. Pinnaduwage, H.-F. Ji, and T. Thundat, "Moore's Law in Homeland Defense: An Integrated Sensor Platform Based on Silicon Microcantilevers," IEEE Sensors Journal, vol. 5, n4, Aug. 2005, pp. 774- 785 .

[6] J. Sader, "Frequency response of cantilever beams immersed in viscous fluids with applications to the atomic force microscope," Journal of Applied Physics, vol 84, Iss 1, 1998, pp. 64-76.

[7] D. Lange, C. Hagleitner, A. Hierlemann, O. Brand, and H. Baltes, "Complementary Metal Oxide Semiconductor Cantilever Arrays on a Single Chip: Mass-Sensitive Detection of Volatile Organic Compounds," Anal. Chem., 74, 2002, pp. 3084-3095.

[8] S. Basak, A. Raman, S. Garimella, "Hydrodynamic loading of microcantilevers vibrating in viscous fluids," Journal of Applied Physics, vol 99, 2006, 114906

[9] I. Dufour, S. Heinrich, and F. Josse, "Theoretical analysis of strongaxis bending mode vibrations for resonant microcantilever (bio)chemical sensors in gas or liquid phase," Journal of MicroElectroMechanical Systems, vol. 16, 2007, pp. 44-49.

[10] U. Lindholm, D. Kana, W.-H. Chu, and H. Abramson, "Elastic vibration Characterisitcs of Cantilever Plates in Water," Journal of Ship Research, vol 9, 1965, pp. 11-22.

[11] J. Ferry, "Viscoselastic Properties of Polymers", $3^{\text {rd }}$ Edition, New York: John Wiley \& Sons, 1980, pp. 606 